\title{
MCMC Particle Filter Using New Data Association Technique with Viterbi Filtered Gate Method for Multi-Target Tracking in Heavy Clutter
}

\author{
E. M.Saad \\ Department of Electronics and Communication Engineering \\ Helwan University \\ Cairo, Egypt \\ H.I.ALI \\ Department of Electronics and Communication Engineering \\ Helwan University \\ Cairo, Egypt
}

\begin{abstract}
Improving data association technique in dense clutter environment for multi-target tracking used in Markov chain Monte Carlo based particle filter (MCMC-PF) are discussed in this paper. A new method named Viterbi filtered gate Markov chain Monte Carlo VFG-MCMC is introduced to avoid track swap and to overcome the issue of losing track to highly maneuvering targets in the presence of more background clutter and false signals. An adaptive search based on Viterbi algorithm is then used to detect the valid filtered data point in each target gate. The detected valid point for each target is applied to the estimation algorithm of MCMC-PF during calculating the sampling weights. This proposed method makes the MCMC interacts only with the valid target that is candidate from the filtered gate and no more calculations are considered for invalid targets. Simulation results demonstrate the effectiveness and better performance when compared to conventional algorithm MCMC-PF.
\end{abstract}

Keywords- data association; multi-target tracking; particle filter; Viterbi algorithm ; Markov chain Monte Carlo; filtering

\section{INTRODUCTION}

In multi-target tracking systems (MTT) data association is an important key to associate the track to the true target in noisy measurements. The measurements (target of interest, clutter, noise signal) are reported by sensor at regular time interval (scan period). The received measurements in a cluttered environment may not originate from the real targets. Some of them may be from clutter or false alarm. As a result, the association between the tracked target and true candidate measurement is difficult. Assigning wrong measurements to track often results in lost track and track breaks. Moreover, in heavy clutter density the resulting number of false tracks may overwhelm the available computational resources of the MTT systems. For this reasons, improving data association technique has received much attention in MTT research [1-6]. The target tracking consists of two basic parts: data association and tracking filtering. Data association is responsible for detecting on each scan which of the received multiple measurements that

\author{
El. Bardawiny \\ Department of Radar \\ MTC College \\ Cairo, Egypt
}

\author{
N.M.Shawky \\ Department of Electronics and Communication Engineering \\ Helwan University \\ Cairo, Egypt
}

lie in the specified gates of the predicted targets position should update with the existing tracking targets. Due to data association, each scan takes the output of the gating process [7] and makes final measurement to track associations. A gating process with filtering method based on Viterbi algorithm is used in this part to find the valid measurement that is considered as the best candidate measurement originated from the true target and assume the remaining measurements to be invalid that originated from false alarm or clutter. The Viterbi algorithm (VA) [8] is a dynamic programming technique for finding the shortest path through a trellis in a computationally efficient manner. The using of the Viterbi algorithm for solving the data association problem when tracking targets in clutter was first suggested as VA-QF by Quach and Farooq [9]. Their approach finds the approximate maximum likelihood assignment of measurements to the target. The problem they considered was that of tracking a single, maneuvering target assumed to be present in surveillance region. This method was modified in [10] to suit the problem of tracking multiple, non maneuvering targets in heavy clutter and was applied to tracking with an Over-The-Horizon radar (OTHR). Instead of using [9] in data association for single target that deals with multi state (multi-scan) where each state contains all measurements according to the corresponding time scan and is processed with the next scan up to all measurements received in the current scan. A new technique for gating process using Viterbi algorithm for multi-target tracking is introduced to be considered as an extension of [9] to detect the measurement in the current gate of the tracked target that has the maximum cost. This extension method for each track depends only on the measurements in current and previous gate of the same tracked target position by two successive states, each state represent the corresponding time scan for all tracked target gate. The candidate measurements in data association part are applied to tracking filter part for estimation. Due to the track swapping and highly maneuvering target, the mainly considered problem for estimation, traditionally, is solved using linearized filters, such as the extended Kalman filter (EKF) [11-15], under a 
Gaussian noise assumption. The sufficient statistics from the linearized filter are used for data association. When dealing with non-linear models in state equation and measurement relation and a non- Gaussian noise assumption, these estimation methods may lead to non-optimal solutions. The sequential Monte Carlo methods, or particle filters [14-21], provide general solutions to many problems where linearizations and Gaussian approximations are intractable or would yield too low performance. Recently MCMC-based particle filter (MCMC-PF) [22-26] has captured the attention of many researchers in various applications that deal with difficult nonlinear and/or non Gaussian problems for tracking maneuvering target in clutter. In MCMC-PF, the particles are sampled from the target posterior distribution via direct MCMC sampling method, which avoids sample impoverishment. To increase the robustness of the algorithm and tracking a highly maneuvering target without swap in dense clutter environment, we introduce the proposed algorithm named VFG-MCMC. The new technique based on Viterbi algorithm as mentioned above is combined with MCMC-PF. Simulation results showed better performance and more effective at tracking when compared to the conventional MCMC-PF algorithm.

\section{PROBLEM FORMULATION}

In a dynamic state space model, the observed signals (observation/ measurements) are associated with a state and measurement noise. Consider that there are $T$ targets being tracked at time index $k$. The time evolution of the state $x_{t}(k) \in R^{n}$ of a discrete-time, nonlinear, dynamic systems is described by the following equation

$$
x_{t}(k)=f\left(x_{t}(k-1), w_{t}(k-1)\right) \quad t=1,2, \ldots, T
$$

where $f$ is the system transition function and $w_{t}(k-1) \in R^{n}$ is a dynamic noise which has a known probability density function (PDF). The superscript $t$ corresponds to the $t^{\text {th }}$ target. The initial target state, $x_{t}(0)$ for $t=1,2, \ldots, T$, is assumed to be known. At discrete times, the measurements $z(k) \in R^{m}$ of the state $x_{t}(k)$ become available and are related to state through the observation equation

$$
z(k)=h\left(x_{t}(k), v(k)\right) \quad t=1,2 . . T
$$

where $h$ is a nonlinear measurement function and $v(k) \in R^{m}$ is a sequence of observation noises of known PDF. From the Bayesian perspective, the tracking problem is to recursively calculate the posterior probability density function, $p\left(x_{t}(k) \mid z_{1: k}\right)$, where $z_{1: k}=\left\{z_{m}, m=1, \ldots . . k\right\}$.

\section{A. Viterbi Data Association VA-QF for Single Target}

The basis of the VA algorithm is built upon the Markov process [9],[27],[28]. In VA algorithm, the target motion is assumed to be a Markov process. The Markov process uses state diagrams where the nodes or measurements represent the state in each scan and the arcs are represented by transition paths. As time progress, the process will trace some path from state (or scan) to state through the state diagram. If the states and their transitions are repeated in time for $\mathrm{k}=1, . ., \mathrm{K}$, we obtain a trellis that represent a directed graph consisting of a set of measurements and paths as shown in Fig. 1. At each measurement from scan to scan, the length of the paths coming into the measurement is summed. The path with the shortest length will be assigned to that measurement and is considered as survivor path (one survivor for each measurement). the recursion will proceed from first to last (current) scan, once the last measurement is reached, the algorithm summing all the smallest (path metric) nodes from each previous scan to obtain the optimal path. A path is a collection of directed arcs that connects an element at stage 1 to an element at stage $\mathrm{K}$. Each directed arcs is associated with a metric or a distance label $a_{i j}(k)$. A path metric is defined as the sum of the metrics of all the arcs contained in the path as

$$
d(p)=\sum_{k=2}^{K} a_{i j}(k) ; \quad\left\{n_{i}(k-1), n_{j}(k)\right\} \in p
$$

By applying the Viterbi algorithm to the trellis diagram, we obtain the sequence of measurements that maximizes the overall likelihood function, thus solving the data association problem as in the following steps.

1) Initialization step: assign a value of zero to the label at each node/measurement in stage 1:

$$
\begin{gathered}
\text { score }_{i}(1)=0, \quad 1 \leq i \leq n_{1} \\
\psi_{i}(1)=0, \quad 1 \leq i \leq n_{1}
\end{gathered}
$$

2) Repeat the following procedure for each stage $k$, where $k=2, \ldots . K$

- For each node $i=0, \ldots \ldots n_{k-1}$ (at stage $\left.k-1\right)$, calculate the predicted position $\hat{z}_{i}(k \mid k-1)$ conditioned on the measurement sequence termination at the node $n_{i}(k-1)$

- For each node $j=0, \ldots \ldots n_{k}$ (at stage $k$ ), calculate the distance label $a_{i j}(k)$ by calculating the normalized negative log likelihood function ( non -maneuvering target) of the arc joining nodes $n_{i}(k-1)$ and $n_{j}(k)$ as given in [27-29]

$$
\begin{gathered}
a_{i j}^{k}=\left\{\begin{array}{ll}
-\ln \{\Lambda(.)\}, & j \neq 0, i=1, \ldots, n_{k}-1 \\
0 \quad & j=0, i=1, \ldots . n n_{k}-1
\end{array}\right\} \\
-\ln \{\Lambda(.)\}=a_{i j}(k)=\left[0.5\left(\tilde{z}_{i j}(k)\right)^{\prime}\left(s_{i}(k)\right)^{-1}\left(\tilde{z}_{i j}(k)\right)\right] \\
-\ln \left\{\frac{p_{D} V_{s}}{1-p_{D}}\right\}+\ln \left\{\left.\left.(2 \pi)^{n / 2}\right|_{s_{i}}(k)\right|^{0.5}\right\}
\end{gathered}
$$

where the innovation $\tilde{z}_{i j}(k)=z_{j}(k)-\hat{z}_{i}(k \mid k-1)$ 
follow:

- assign node $n_{j}(k)$ with the smallest label as

$$
\begin{aligned}
& d_{j}(k)=\min _{0 \leq i \leq n(k-1)}\left\{d_{i}(k-1)+a_{i j}(k)\right\} \\
& \left.* \quad \min _{0 \leq i \leq n(k-1)}\left\{d_{i}(k-1)+a_{i j}(k)\right)\right\}
\end{aligned}
$$

and let

$$
\begin{gathered}
\operatorname{score}_{j}(k)=\left(d_{i}(k-1)+a_{i j}(k)\right) \\
* \\
\psi_{j}(k)=i
\end{gathered}
$$

- update the target state $\hat{x}_{j}(k)$ and its covariance $p_{j}(k)$ at each node in stage $\mathrm{k}$

3) Final selection: determine the node with the minimum score in the final stage. The current state of the target is the state associated with the minimal node.

$$
\begin{gathered}
i=\arg \left\{\min _{0 \leq i \leq n_{K}}\left\{\text { score }_{i}(k)\right\}\right\} \\
\hat{x}(K)=\hat{x}_{i}^{*}(K)
\end{gathered}
$$

4) Back track: recover the measurement sequence that terminates with the minimum node score in the final stage using

$$
\left.{ }_{i}^{*}\right|_{k-1} ^{*}=\psi_{i}^{*}(k)
$$

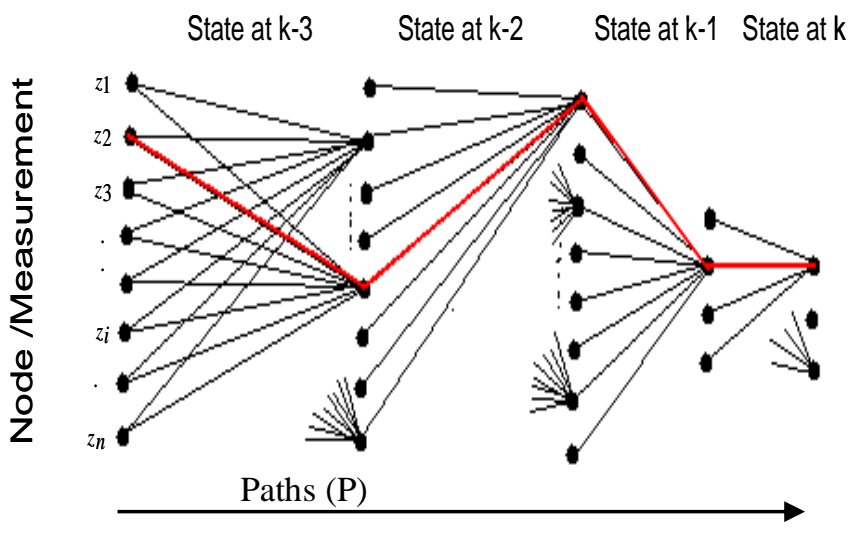

Figure 1. Trellis Diagram of VDA for tracking target

\section{B. Markov Chain Monte Carlo based Particle Filter(MCMC $-P F)$}

\section{1) Basic theory of particle filter}

The particle filter is a recent filtering method based on Bayesian estimation and Monte Carlo method and effectively deals with complicated nonlinear and /or non-Gaussian problems. The basic idea of this method is Monte Carlo simulation, in which the posterior density is approximated by a set of particles with associated weights $\left\{\left(x_{t}^{i}(k-1), w_{t}^{i}(k-1) \quad i=1,2, . . N\right\}\right.$.

At every time step we sample from the proposal distribution $q\left(x_{t}(k) / x_{t}(k-1), z 1: k\right)$ to achieve new particles and compute new weights according to the particle likelihoods. After normalization of weights, the posterior density can be represented by $\left\{\left(x_{t}^{i}(k), w_{t}^{i}(k) \quad i=1,2, \ldots N\right\}\right.$.

The implementation of the basic particle filter is as follows:

(1) Initialization

Draw a set of particles from the prior $p\left(x_{t}(0)\right)$ :

$$
x_{t}^{i}(0) \sim p\left(x_{t}(0)\right), \quad i=1,2 \ldots, N
$$

\section{Prediction:}

(2) Sampling step

(a) for $i=1,2, \ldots N$

Sample $x_{t}^{i}(k)$ from the proposal distribution $q\left(x_{t}(k) \mid x_{t}(k-1), z 1: k\right)$ :

$x_{t}^{i}(k) \sim q\left(x_{t}(k) \mid x_{t}(k-1), z 1: k\right)$

\section{Update:}

(b) Evaluate the importance weights when the candidate measurement $z(k)$ is choosing by data association algorithm

$$
w_{t}^{i}(k)=w_{t}^{i}(k-1) \frac{p\left(z(k) \mid x_{t}^{i}(k)\right) p\left(x_{t}^{i}(k) \mid x_{t}^{i}(k-1)\right.}{q\left(x_{t}(k) \mid x_{t}(k-1), z 1: k\right)}
$$

(c) Normalize the weights

$$
\tilde{w}_{t}^{i}(k)=w_{t}^{i}(k) / \sum_{i=1}^{N} w_{t}^{i}(k) \quad, i=1,2 \ldots, N
$$

(3) Markov Chain Monte Carlo (MCMC) step as described in algorithm 1

(4) Output step

Output a set of particles $\left\{\left(x_{t}^{i}(k), w_{t}^{i}(k) \quad i=1,2, \ldots N\right\}\right.$ that can be used to approximate the posterior distribution.

Expectation:

$$
\hat{x}_{t}(k)=\sum_{i=1}^{N} \tilde{w}_{t}^{i}(k) x_{t}^{i}(k)
$$

Covariance:

$$
p_{t}(k)=\sum_{i=1}^{N} \tilde{w}_{t}^{i}(k)\left(x_{t}^{i}(k)-\hat{x}_{t}(k)\right)\left(x_{t}^{i}(k)-\hat{x}_{t}(k)\right)^{\prime}
$$


and set $w_{t}^{i}(k)=1 / N, \quad i=1,2, \ldots N$

(5) $k=k+1$, go to step 2

\section{2) Basic theory of Markov Chain Monte Carlo (MCMC)}

The MCMC step, as described in [24] has an invariant

distribution $\prod_{i=1}^{N} P\left(x_{t}^{i} 0: k \mid z 1: k\right)$, which is applied to each of the $N$ particles, one at the time.

The metropolis- Hastings algorithm $(\mathrm{MH})$ is a way to simulate from such a chain. The idea of $\mathrm{MH}$ is to sample states from a Markov Chain with the posterior as invariant distribution.

A Markov Chain is constructed by approximate a candidate for the next state $x_{t}^{* i}(k)$ given the current state $x_{t}^{i}(k)$ according to the proposal distribution $p\left(x_{t}(k) \mid x_{t}(k-1)\right)$. This state transition is accepted with probability

$\alpha\left(x_{t}^{i}(k), x_{t}^{* i}(k)\right)=\min \left\{1, \frac{p\left(z(k) \mid x_{t}^{* i}(k)\right.}{p\left(z(k) \mid x_{t}^{i}(k)\right.}\right\}$

\section{Algorithm 1: Markov Chain Monte Carlo (MCMC) Step}

(a) For each particle 1:N sample the proposal candidate for the approximation of the next state.

$$
x_{t}^{* i}(k) \sim p\left(x_{t}(k) / x_{t}(k-1)\right)
$$

(b) Sample $\rho \sim \mathrm{U}(0,1)$, where $\mathrm{U}(0,1)$ is a uniform distribution in the interval $(0,1)$.

(c) If $\rho \leq \min \left\{1, \frac{p\left(z(k) \mid x_{t}^{*} i(k)\right.}{p\left(z(k) \mid x_{t}^{i}(k)\right.}\right\}$

then accept move:

$$
x_{t}^{i}(k)=x_{t}^{* i}(k)
$$

else reject move

$$
x_{t}^{i}(k)=x_{t}^{i}(k)
$$

end if.

\section{VITERBI FILTERED GATE DATA ASSOCIATION}

To introduce a robust filtering technique with improving tracking performance and give the tracking system the capability to track the multi-target when moving with highly maneuvering without swapping and in dense clutter environment, a new algorithm, named Viterbi filtered gate based Markov Chain Monte Carlo- particle filter (VFGMCMC) as shown in Fig. 2, is proposed.
In the prediction step, Let $Z(k-1)=\left\{z_{1}(k-1), z_{2}(k-1), \ldots z_{w_{n}}(k-1)\right\}$ be a set of points in the 2-D Euclidean space at time $k-1$ where $w_{n}$ is the number of points at time scan $\Delta t$ and let $\hat{z}^{t}(k)$ be a predicted position of the $t^{\text {th }}$ tracked target at time $k$. according to distance metric measure and gate size, let $\bar{Z}^{t}(k-1)=\left\{z_{1}(k-1), . . z_{i}(k-1), . . z_{m_{i}}(k-1)\right\}$ be a set of the candidate points detected in the $t^{\text {th }}$ gate $G_{t}(k-1)$ of predicted position $\hat{z}^{t}(k)$ whose elements are a subset from the set $Z(k-1)$ where $i=1$ to $m i$ ( number of detected points in gate $G_{t}(k-1)$ at time $\left.k-1\right)$ and $\bar{Z}^{t}(k-1)$ be a set of all valid points $z_{i}(k-1)$ that satisfy the distance measure condition $\left(z_{i}(k-1)-\hat{z}^{t}(k)\right)^{\prime} S^{t}(k)^{-1}\left(z_{i}(k-1)-\hat{z}^{t}(k)\right) \leq \gamma$

for each target $t$ where $\gamma$ is threshold value that determines the gate size and $l=1$ to $w_{n}, i=1$ to $m i$, i. e for each target $t, i$ is initialized by 1 and is increased by $i=i+1$ after each valid point is detected up to last $m i$ detected points.

In the updating step, let $Z(k)=\left\{z_{1}(k), z_{2}(k), \ldots z_{w_{c}}(k)\right\}$ be a set of points in the 2-D Euclidean space at time $k$ where $\boldsymbol{w}_{\boldsymbol{C}}$ is the number of points at time scan $\Delta t$. The candidate points detected in the same gate $G_{t}(k)$ as $G_{t}(k-1)$ of the $t^{\text {th }}$ predicted position $\quad \hat{z}^{t}(k)$ is a subset $\bar{Z}^{t}(k)=\left\{z_{1}(k), . . z_{j}(k), . . z_{m j}(k)\right\} \quad$ from the set $Z(k)$ where $j=1$ to $m j$ (number of detected points in $t^{\text {th }}$ gate at time $k$ ) and $\bar{Z}^{t}(k)$ be a set of all valid points $z_{j}(k)$ that satisfy the distance measure condition $\left(z j(k)-\hat{z}^{t}(k)\right)^{\prime} S^{t}(k)^{-1}\left(z j(k)-\hat{z}^{t}(k)\right) \leq \gamma$

for each target $t$ where $l=1$ to $w_{\boldsymbol{C}}, j=1$ to $m j$ for $j=j+1$ after each valid point is detected. A new filtering gate method based Viterbi algorithm is proposed to distinguish between the detected measurements in $G_{t}(k)$ that originated from the target or originated from clutter ( false target ).

In this method we consider the two constructed gate $G_{t}(k-1), G_{t}(k)$ for each target as the two successive state as mentioned above in viterbi algorithm VA-QF for single target. This method is based only on the measurements (nodes) $\left\{z_{i}(k-1), z_{j}(k)\right\}$ that fall in the two previous and current gate $\left\{G_{t}(k-1), G_{t}(k)\right\}$ for each target that have the same predicted target position at time scan $k-1, k$ respectively. 


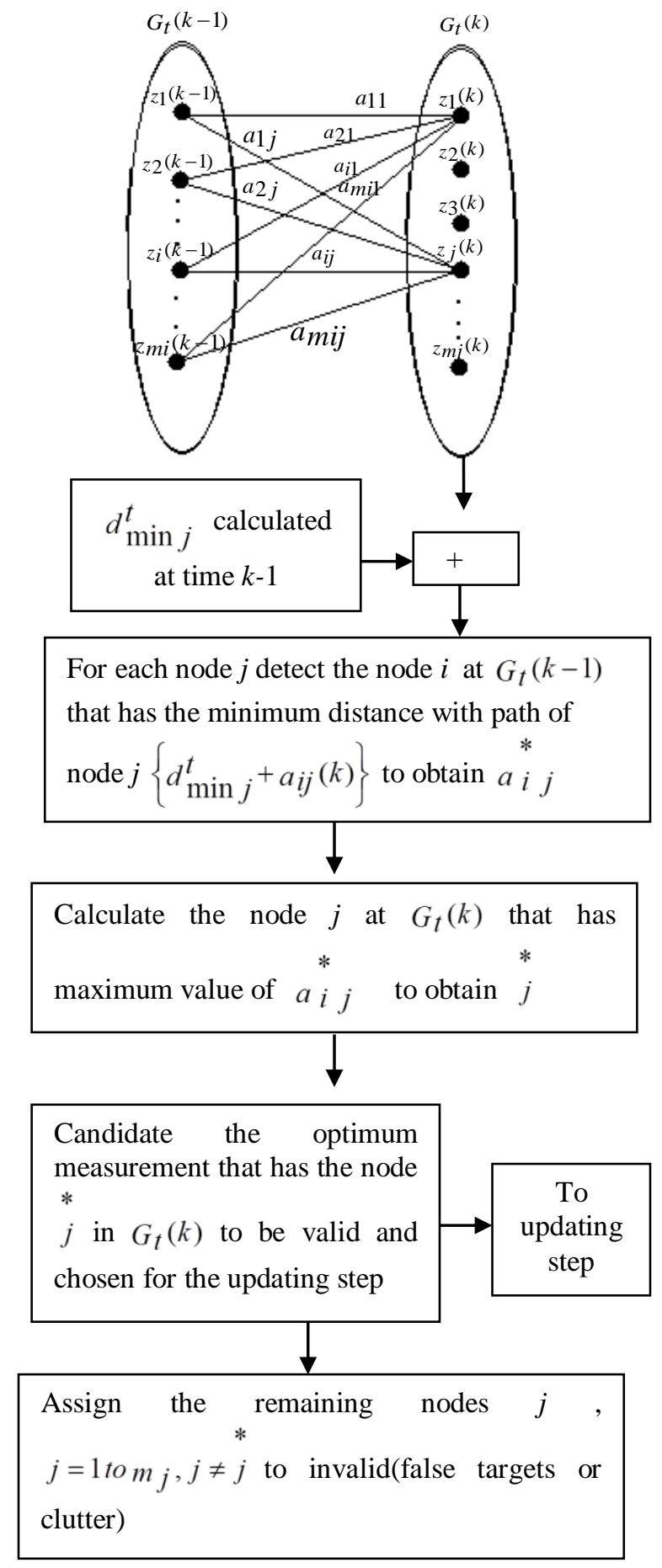

Figure 2. Schematic Diagram of VFG data association algorithm

To detect the candidate valid measurement $z_{j}^{*}(k)$ from the set $\bar{Z}^{t}(k)$ of the $t^{\text {th }}$ gate $G_{t}(k)$ to be considered as the valid measurement that originate from the target and as the choosing measurement in the updating step, we first calculate distance metric between each measurement $z j(k)$ in current gate $G_{t}(k)$ and all measurements $z_{i}(k-1)$ in its previous gate $G_{t}(k-1)$ as given

$$
\begin{aligned}
a_{i j}^{k}= & \left\{\begin{array}{ll}
-\ln \{\Lambda(.)\}, & j=1, \ldots m_{j}, i=1, \ldots . m_{i} \\
0 & j=0, i=1, \ldots . m_{i}
\end{array}\right\} \\
-\ln \{\Lambda(.)\}= & a_{i j}(k)=\left[0.5\left(\tilde{z}_{i j}(k)\right)^{\prime}\left(s_{i}(k)\right)^{-1}\left(\tilde{z}_{i j}(k)\right)\right] \\
& -\ln \left\{\frac{p_{D} V_{s}}{1-p_{D}}\right\}+\ln \left\{(2 \pi)^{n / 2}\left|s_{i}(k)\right|^{0.5}\right\}
\end{aligned}
$$

where the innovation in this method is calculated as $\tilde{z}_{i j}(k)=z_{j}(k)-z_{i}(k-1)$

The minimum distance $d_{\min j}^{t}$ that represents the shortest path at each measurement $z_{j}(k)$ is assigned to its corresponding distance metric $a_{i j}(k)$ to give

$$
a^{+}{ }_{i j}(k)=d_{\min j}^{t}+a_{i j}(k), \quad j=1, \ldots m_{j}, i=1, \ldots, m_{i}
$$

For each measurement label $j$ in $G_{t}(k)$, we choose one of the path metric $a^{+}{ }_{i j}(k), i=1, \ldots . m_{i}$ that has the minimum distance and then its measurement label (index) $i$ in $G_{t}(k-1)$ is obtained by

$$
\left.\begin{array}{l}
\begin{array}{l}
* \arg \left\{\min _{1 \leq i \leq m_{i}}\{a+i j(k)\}\right\} \\
* \\
\text { score } j \\
d_{\min j}^{t}=a_{i j}^{t} \\
\min j \\
*
\end{array} a_{i j}
\end{array}\right\} j=1, \ldots m_{j}
$$

After determining the shortest path with minimum distance $a_{i j}^{*}$ for each measurement label $j$ in $G_{t}(k)$, the valid measurement $j$ that is a candidate to be the associating measurement to a tracked target $t$ in the updating step is calculated by obtaining the measurement index label of the maximum score $_{j}(k)$ as in the following equation

$$
j=\arg \max _{1 \leq j \leq m_{j}} \text { score }_{j}(k)
$$


Thus, for each target $t$ at each new scan, the filtered gate using this proposed method of data association technique select one measurement from the target gate that is considered to be valid (originate from the true target) and is assigned to be the selected or candidate measurement used in the process for estimation in the updating step, while the remaining measurements are considered to be invalid (originate from clutter) and are avoided from tracking process.

\section{IMPLIMENTATION OF VITERBI FILTERED GATE DATA ASSOCIATION WITH MCMC-PARTICLE FILTER (VFG- MCMC)}

The proposed algorithm VFG-MCMC is an approximate likelihood- based approach for solving the data association problem in multi target tracking when the clutter is heavy. This new algorithm depends on the history of measurements for one scan and the construction current gate. The current gate of the tracked target is processed with the previous gate of the same tracked target position using an advanced technique based on the basic principle of viterbi algorithm VA-QF. This technique detects the one valid measurement in the current gate that is assumed to be originated from true target, while the remaining measurements in the gate are considered as invalid that are assumed to be originated from clutter or false target. This filtering gate method select or candidate the one valid measurement for each target to be processed in the updating step while the invalid measurements not include in the tracking process. The proposed data association algorithm is combined with MCMC-PF to overcome the problem of highly maneuvering target and swapping in the presence of dense clutter. The proposed algorithm VFG using MCMC-PF is represented in algorithm 2.

\section{Algorithm 2: VFG-MCMC}

\section{For $t=1$ to $T$}

\section{Initialization}

1- Draw a set of particles from the prior $p\left(x_{t}(0)\right)$ : $x_{t}^{i}(0) \sim p\left(x_{t}(0)\right), \quad i=1,2 \ldots, N \quad, \quad w_{t}^{i}(0)=1 / N$

\section{Prediction:}

2- With sampling step Particles at time step $k-1,\left\{\hat{x}_{t}^{i}(k-1)\right\}$, are passed through the dynamic state model as in (1) to obtain the predicted particles

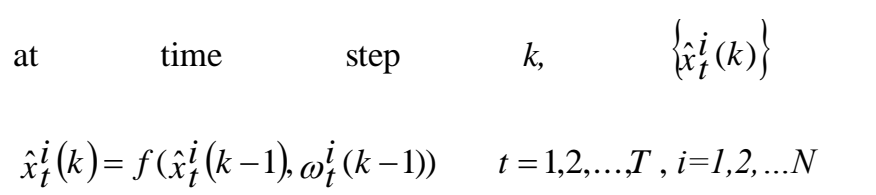

3- Without sampling step
(a)Expectation at time step $k-1,\left\{\hat{x}_{t}(k-1)\right\}$, are passed through the dynamic state model as in (1) to obtain the predicted particles at time step $k, \quad\left\{\hat{x}_{t}(k)\right\}$ :

$\hat{x}_{t}(k)=f\left(\hat{x}_{t}(k-1), \omega_{t}(k-1)\right) \quad t=1,2, \ldots, T$

$\hat{z}^{t}(k)=h\left(\hat{x}_{t}(k), \quad t=1,2 . . T\right.$

(b) Finding validate measurement $z(k)=z_{j}^{*}(k)$ according to algorithm 3

\section{Update:}

4- Once the measurement data $z(k)$ is obtained by data association algorithm VFG, evaluate the importance weight of each predicted particle

$$
w_{t}^{i}(k)=w_{t}^{i}(k-1) p\left(z(k) \mid \hat{x}_{i}^{i}(k)\right)
$$

and normalize the weights

$$
\tilde{w}_{t}^{i}(k)=w_{t}^{i}(k) / \sum_{i=1}^{N} w_{t}^{i}(k) \quad, i=1,2 \ldots, N
$$

5- Markov Chain Monte Carlo (MCMC) step as described in algorithm 1

\section{6- Output step}

Output a set of particles $\left\{\left(x_{t}^{i}(k), w_{t}^{i}(k) \quad i=1,2, . . N\right\}\right.$ that can be used to approximate the posterior distribution by Calculating the expectation $\hat{x}_{t}(k)$ and Covariance $p_{t}(k)$ using (16),(17) respectively

and set $w_{t}^{i}(k)$ as in (18)

$7-k=k+1$, go to step 2

8- End for

Algorithm 3: Find the validate measurement using VFG algorithm

1. Find validated region for measurements at time $k-1$ :

$$
\bar{Z}^{t}(k-1)=\left\{z_{i}(k-1)\right\}, \quad i=1, \ldots m i
$$

By accepting only those measurements that lie inside the gate $t$ :

$$
\begin{aligned}
& \bar{Z}^{t}(k-1)=\left\{Z:\left(z_{i}(k-1)-\hat{z}^{t}(k)\right)^{\prime} S^{t}(k)^{-1}\right. \\
& \left.\left(z_{i}(k-1)-\hat{z}^{t}(k)\right) \leq \gamma \quad\right\}
\end{aligned}
$$


2. Find validated region for measurements at time $k$ :

$$
\bar{Z}^{t}(k)=\{z j(k)\}, \quad j=1, . . m j
$$

By accepting only those measurements that lie inside the gate $t$

$$
\begin{aligned}
& \bar{Z}^{t}(k)=\left\{Z:\left(z j(k)-\hat{z}^{t}(k)\right)^{\prime} S^{t}(k)^{-1}\right. \\
& \left.\left(z j_{j}(k)-\hat{z}^{t}(k)\right) \leq \gamma\right\}
\end{aligned}
$$

3. For each measurement $j$ the distance metric $a_{i j}^{k}$ is calculated by (22) using (23), (24)

4. The distance path $a^{+}{ }_{i j}(k)$ is obtained by (25)

5. For each measurement $j$ finds the label measurement $i$ as calculated in (26) that has the minimum distance $a_{i} j$

6. Find the valid measurement $j$ that has the maximum score Using (27).

7. The associated measurement $z(k)$ is set to

$$
z(k)=z_{j}^{*}(k)
$$

Finally, by using VFG data association algorithm, we obtain for each target the valid measurement in the tracked target gate that is applied to MCMC-PF before entering in the process of the updating step. The other measurements in the gate are assumed to be invalid and the updating process for tracking the targets is not assigned to any one of them. VFG data association algorithm has an advantage that is accepted by MCMC-PF to improve the performance in heavy clutter for the tracked targets and to maintain the tracking to the targets that move with high maneuvering and swapping.

\section{Simulation Results}

In order to demonstrate the performance of the proposed VFG-MCMC algorithm, we provide a comparison with the most popular nonlinear conventional tracking algorithm MCMC-PF. Two test scenarios have been chosen in this section for moving more than one target in the XY plane with different issues according to the two different tracking problems; high maneuvering and swapping in dense clutter environment. The discrete time state equation with sampling $\begin{array}{lllll}\text { interval } \Delta t & = & 4 & \mathrm{sec} & \text { is }\end{array}$

$$
x_{t}(k)=F x_{t}(k-1)+\Gamma w_{t}(k-1) \quad t=1,2, . T
$$

where $\left.x_{t}(k)=[x(k), y(k), \& k), \&(k)\right]$ is the state vector ; $x(k)$ and $\& k)$ are respectively the position and velocity of the moving object along the Cartesian frame $\mathrm{X}$ axis; and $y(k)$, $\& k)$ along the $\mathrm{Y}$ axis. $\Gamma$ is a unity matrix and $w_{t}(k-1)$ is discrete time white, Gaussian noise: $w(k) \sim N\left(0, Q_{k}\right)$, $Q=G G^{\prime}$. The measurements are received from one sensor which is positioned at the origin of the plane. The measurement equation is as follow:

$$
z(k)=h\left(x_{t}(k)\right)+v(k) \quad t=1,2 . . T \quad, \quad \text { where }
$$
$z(k)=\left[z_{1}(k), z_{2}(k)\right]$ is the observation vector. $z_{1}(k)$ is the distance between the origin and the moving target and $z_{2}(k)$ is the bearing angle. The measurement noise $v(k)=\left[v_{z 1}(k), v_{z 2}(k)\right]$ is a zero mean Gaussian white noise process with variance $\mathrm{R}$, where $R=\left[\begin{array}{ll}\sigma_{z 1}^{2} & 0 \\ 0 & \sigma_{z 2}^{2}\end{array}\right]$,

$$
\begin{gathered}
z(k)=\left[\begin{array}{l}
\sqrt{\left(x(k)-x_{r}\right)^{2}+\left(y(k)-y_{r}\right)^{2}}+v_{z 1}(k) \\
\arctan \left(\frac{y(k)-y_{r}}{x(k)-x_{r}}\right)+v_{z 2}(k)
\end{array}\right] \text { where } \\
\left(x_{r}, y_{r}\right) \text { is the position of the sensor at the origin, } \\
F=\left[\begin{array}{cccc}
1 & 0 & \Delta t & 0 \\
\frac{\Delta t^{2}}{2} & 0 \\
0 & 1 & 0 & \Delta t \\
0 & 0 & 1 & 0 \\
0 & 0 & 0 & 1
\end{array}\right] G=\left[\begin{array}{ll}
0 & \frac{\Delta t}{2} \\
0 & 0
\end{array}\right]
\end{gathered}
$$

The first test scenario has three tracks with initial state $x_{1}(\mathrm{O})=[13.4 \mathrm{~km}, 7.15 \mathrm{~km}, 200 \mathrm{~m} / \mathrm{s}, 200 \mathrm{~m} / \mathrm{s}], x_{2}(\mathrm{O})=$ $[16.1 \mathrm{~km}, 12.3 \mathrm{~km}, 200 \mathrm{~m} / \mathrm{s}, 200 \mathrm{~m} / \mathrm{s}], x_{3}(\mathrm{O})=[18.3 \mathrm{~km}$, $9.09 \mathrm{~km}, 200 \mathrm{~m} / \mathrm{s}, 200 \mathrm{~m} / \mathrm{s}]$, which continues from the first frame to the last frame. This scenario is used to evaluate the tracking performance by the proposed and conventional algorithm when the targets move with high maneuvering in the presence of heavy clutter. The second test scenario has two tracks with initial state $x_{1}(O)=[13.4 \mathrm{~km}, 12.5 \mathrm{~km}, 150 \mathrm{~m} / \mathrm{s}, 150 \mathrm{~m} / \mathrm{s}]$, $x_{2}(\mathrm{O})=[13.3 \mathrm{~km}, 10.5 \mathrm{~km}, 150 \mathrm{~m} / \mathrm{s}, 150 \mathrm{~m} / \mathrm{s}]$ moving in cross trajectory corrupted by more background of clutter to evaluate 
the tracking performance against swapping between tracks for the two approaches that are presented here. We initiate the other parameters as; the row and column sizes of the volume ( $\left.V_{S}=S_{W} \times S_{H}\right), V_{S}=20 \times 20, \quad T=38 * 4=152 \mathrm{sec}, p_{D}$
$=0.99$, in addition to $R=\left[\begin{array}{ll}400 m^{2} & 0 \\ 0 & 1 \mathrm{deg}\end{array}\right]$, the particle number $N=200$, sensor position $\left(x_{r}, y_{r}\right)=(0,0)$

According to the first test scenario, using the conventional MCMC-PF algorithm in clear environment with no clutter to track highly maneuvering targets, continuing to tracking with no failure as expected as shown in Fig.3 (a). The X-Y trajectory is implemented in Fig 4(a). But this algorithm at low signal to noise ratio fails to track the corrupted targets with a uniform high clutter density as shown in Fig. 3(b) while, given a fixed threshold ( $\gamma=10^{-4}$ ), we showed that the proposed VFG-MCMC succeeded to track the targets in the same environment as shown in Fig. 3(c). We obtain trajectories for $\mathrm{X}$ - and Y- components as shown in Fig. 4(b),(c). In these figures, the colored solid line represents the underlying truth targets of the trajectory (each target with different color) while the colored + symbol represents trajectory of the estimated tracked targets. Our proposed algorithm (+ symbol with different color) detects and associates the proper sequence of observation very well compared to MCMC-PF which fails to continue. According to the second test scenario, the conventional algorithm in clear environment with no clutter fails to avoid swapping between tracks as shown in Fig. $5(a),(b),(c),(d)$ that are represented in different time scan and also its X- Y- trajectory is implemented as shown in Fig. 6 . Thus, when using the conventional algorithm for tracking the targets in more background clutter we fail to track the targets with no avoiding the swapping of the crossed targets as shown in Fig. 7(a). The proposed algorithm that is evaluated in the same clutter environment succeeded to track the targets with no swapping as shown in Fig.7 (b). The X- Y-trajectories for the two approaches to demonstrate the swapping effect and the capability to maintain the tracks in more background clutter are shown in Fig. 8 (a),(b). According to the first and second test scenario we also compared error root mean square value (RMSE) for the different two approaches with three targets and with two targets as shown in Fig. 9,10. Our proposed algorithm in dense clutter environment has lower error, RMSE values and less sensitive to clutter than MCMC-PF over frame numbers.

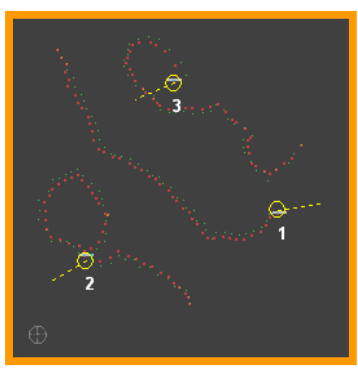

(a)

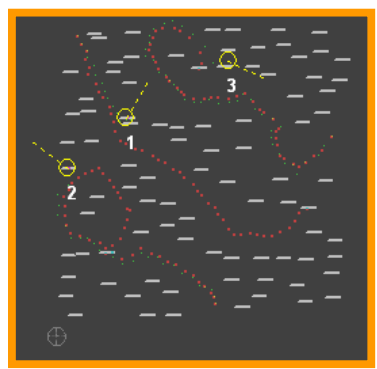

(b)

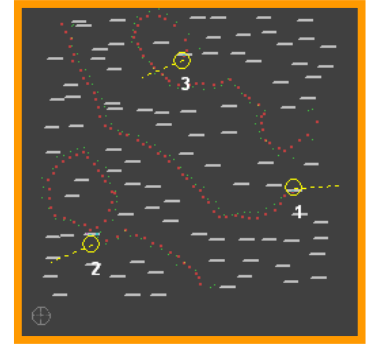

(c)

Figure 3. The state of tracking multi-targets (3 moving targets) using the 2 approaches (a) MCMC-PF succeeded to track in clear environment (no clutter) (b) MCMC-PF failed to continue tracking in heavy clutter (c) VFG-

MCMC succeeded to continue tracking in the same environment as in (b)

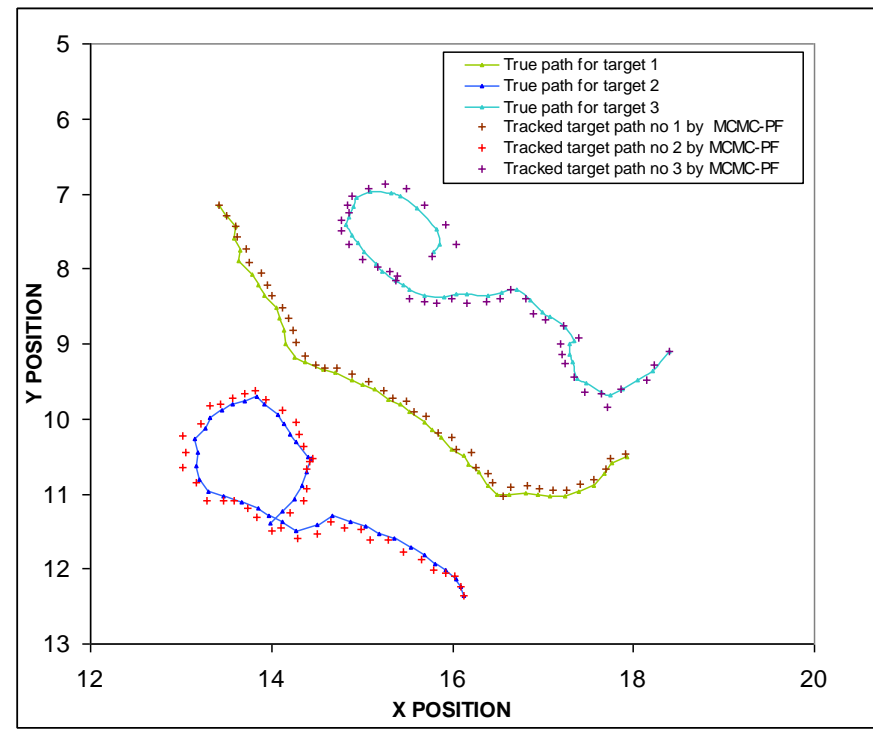

(a)

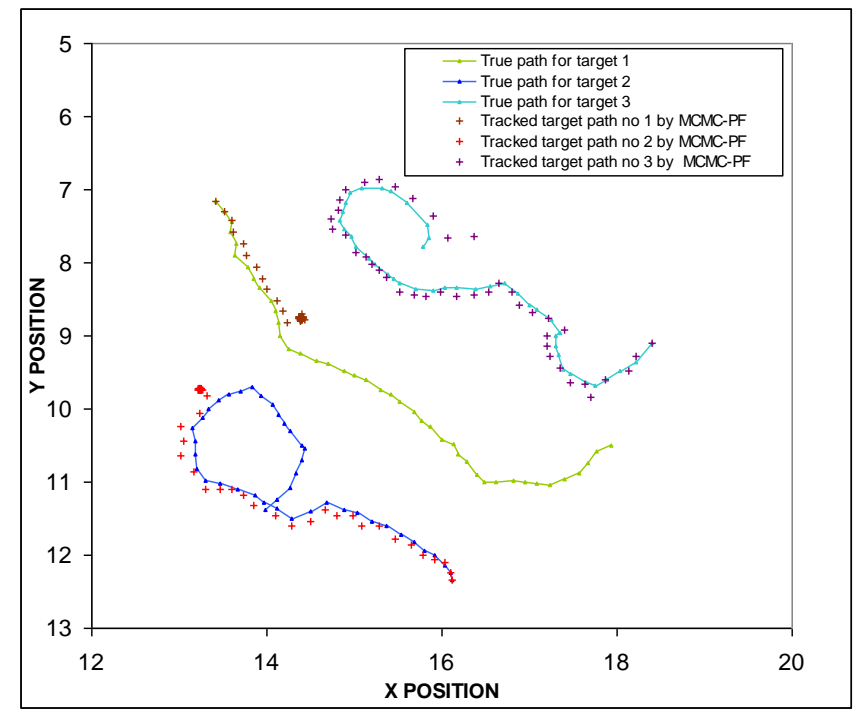

(b) 


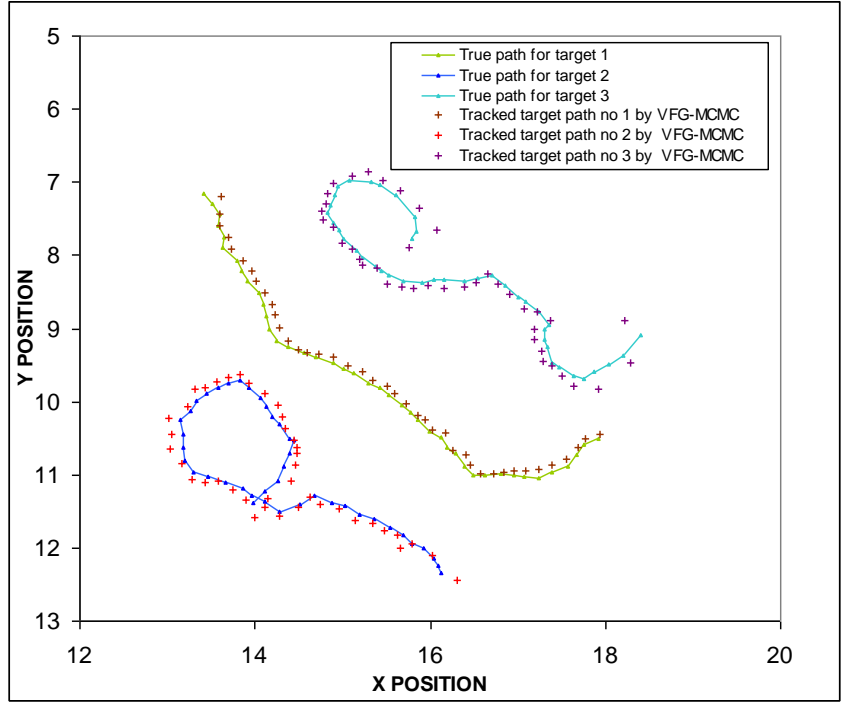

(c)

Figure 4. Trajectory for X-Y components for the MCMC-PF used in tracking 3 targets (+ symbol) without background clutter as in (a) and for the 2 approaches algorithm with heavy clutter as in (b),(c). all showed the true targets path as in solid line. (b) Trajectory for X-Y by MCMC-PF. (c) Trajectory for X-Y by VFG-MCMC.

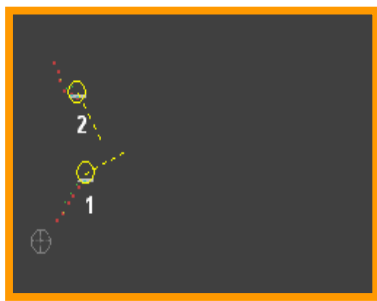

(a)

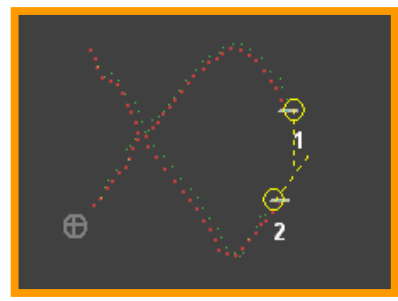

(c)

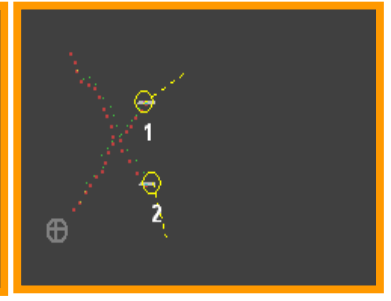

(b)

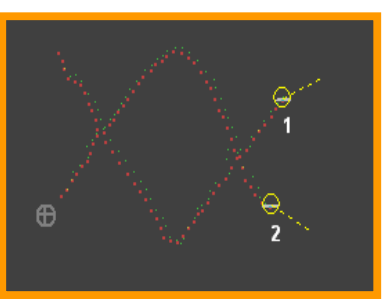

(d)
Figure 5. The swapping state for tracking 2 moving targets in cross trajectory using MCMC-PF at different time scan with no clutter as in (a),(b),(c),(d) This show the track no. 1 swap with track no..2 as shown in (d)

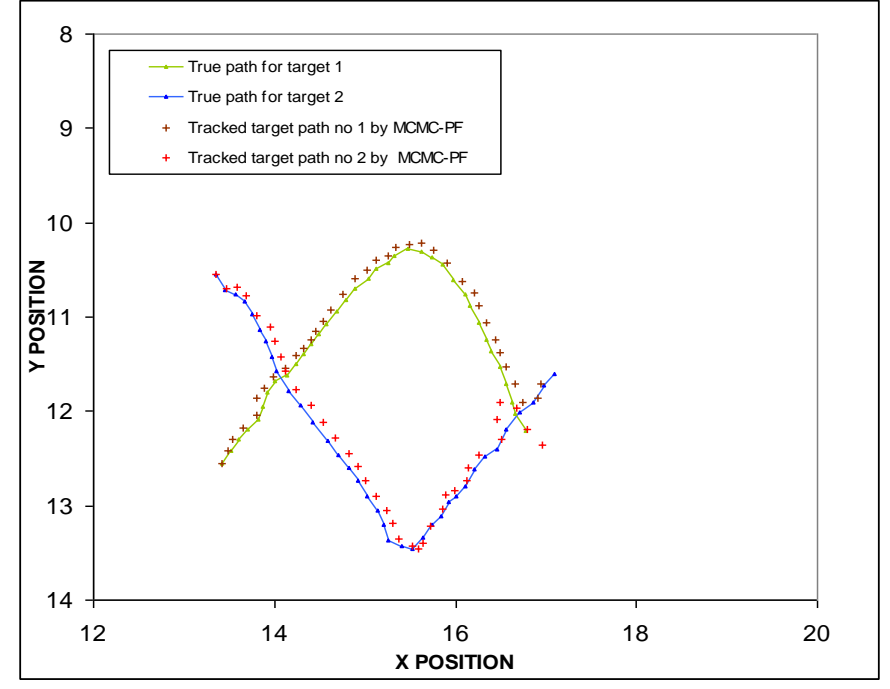

Figure 6. X-Y Components Trajectory for the MCMC-PF used in tracking 2 targets (+ symbol) without background clutter and the true targets Path in solid line. This showed the MCMC-PF fails to avoid swapping.

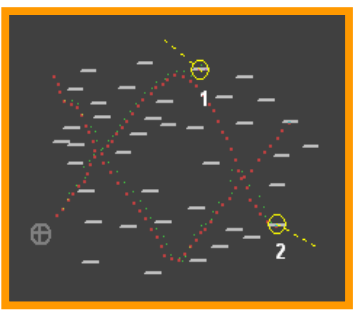

(a)

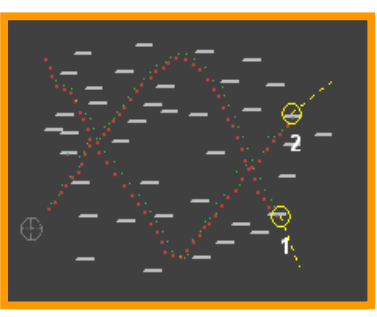

(b)
Figure 7. The swapping state for tracking 2 moving targets in cross trajectory using the 2 approaches in more background clutter (a) MCMC-PF fails to continue to track no 1 and take the wrong trajectory for track no 2 (b) VFG- MCMC succeeded to continue tracking and avoided swapping .

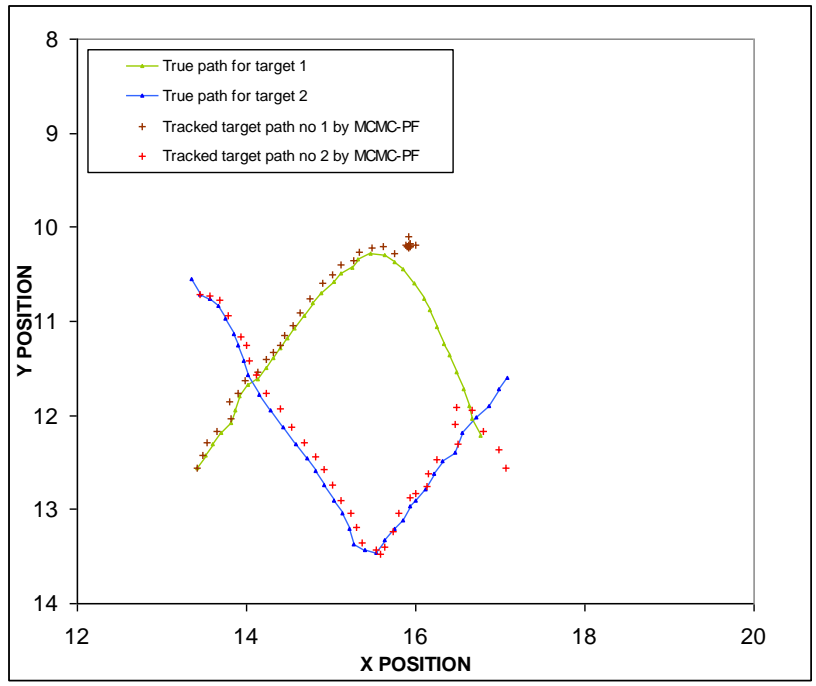

(a) 


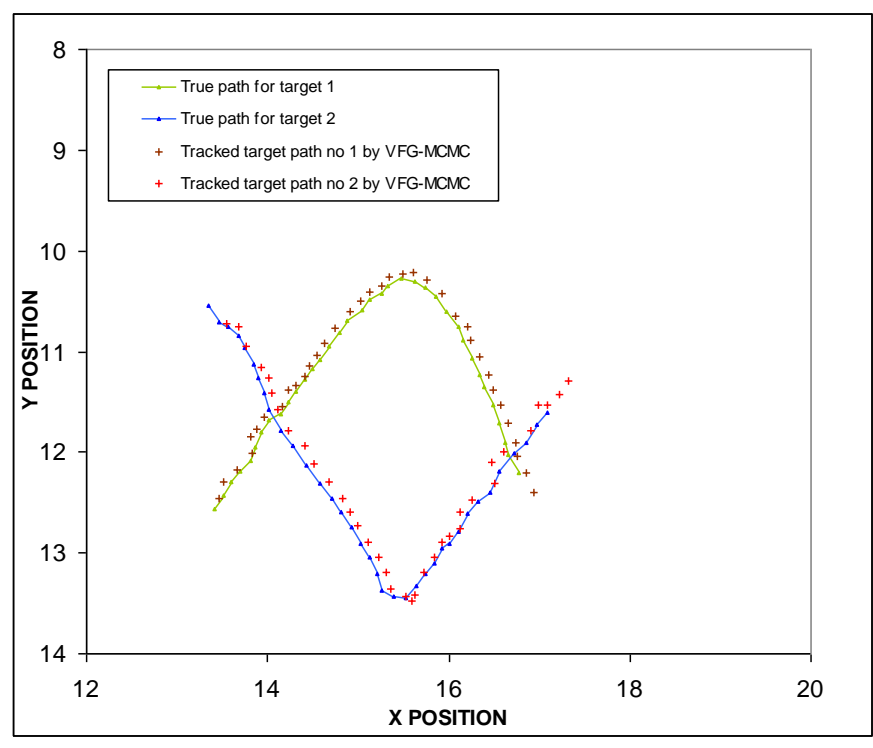

(b)

Figure 8. X-Y Components Trajectory for the 2 approaches algorithm used in tracking 2 targets (+ symbol) in more background clutter and showed the true target path as in solid line. (a) Trajectory for X-Y by MCMC-PF (b) Trajectory for X-Y by VFG-MCMC.

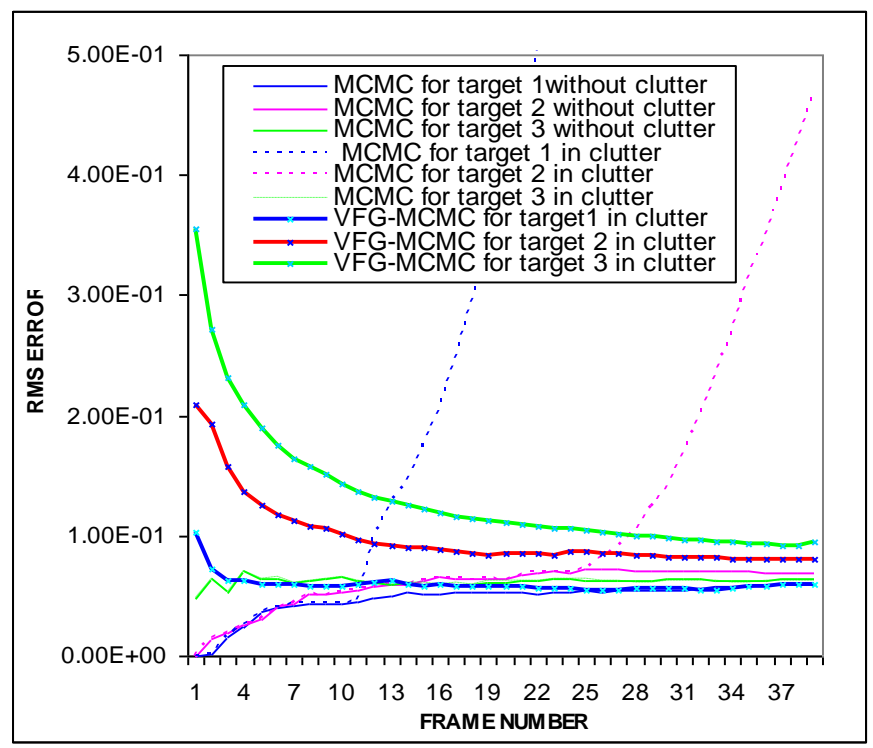

Figure 9. The RMSE for each target (3 targets) separately over frame number (each frame take $4 \mathrm{sec} /$ one scan) according to tracking evaluation of the first test scenarios as shown in Fig. 3,4 .

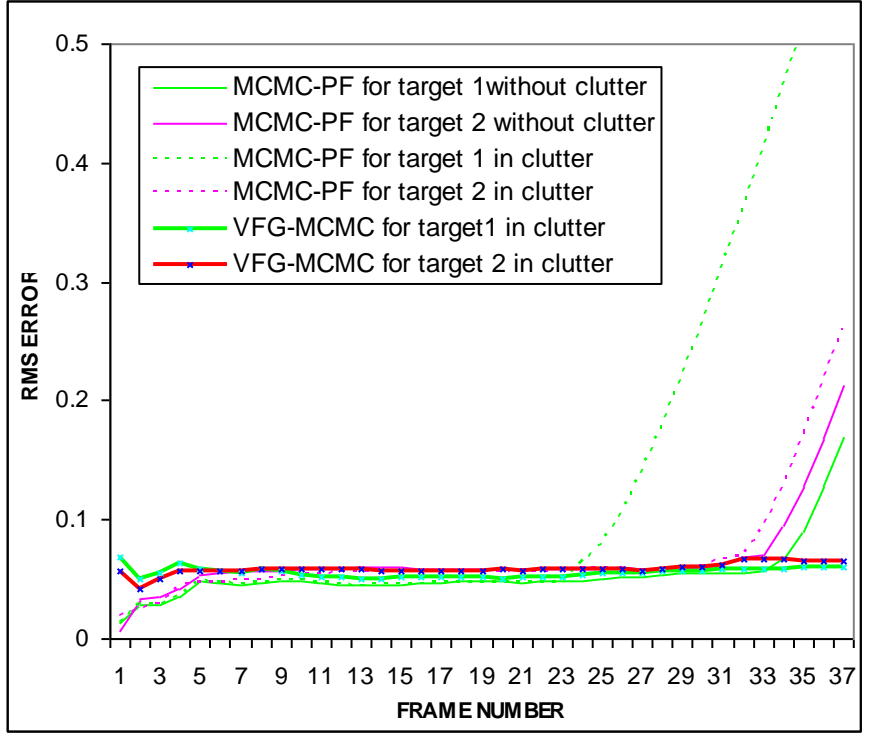

Figure 10. The RMSE for each target (2 targets) separately over frame number (each frame take $4 \mathrm{sec} /$ one scan) according to tracking evaluation of the second test scenarios as shown in Fig. 5,6,7,8.

\section{CONCLUSION}

In this paper for multi-target tracking in dense clutter environment, a new data association technique based on filtering gate method using Viterbi algorithm (VFG) applied to MCMC-PF, can solve the tracking problem effectively, such as ; highly maneuvering target, track swapping. From the results obtained in the simulations, we have showed that in dense clutter environment, the MCMC-PF fails to tracks the high maneuvering targets and fails to avoid track swapping for the moving crossed target trajectory, where the proposed Viterbi filtered gate (VFG-MCMC) algorithm is capable of tracking the targets and avoid swapping. The VFG-MCMC avoids the false targets from the valid based measurement regions and chooses the best candidate valid measurement to the tracking filter MCMC-PF using VFG algorithm. Thus, this improves the data association process which has been shown to give targets the ability to continue to be tracked in dense clutter and also improves the tracking performance of MCMC-PF. This approach can be used to overcome the clutter of gate based approaches in tracking.

\section{REFERENCES}

[1] Y. Bar-Shalom and T. E. Fortmann, "Tracking and data association", academic press, 1988.

[2] Yaakov Bar-Shalom, Fred Daum, and Jim Huang. "The probabilistic 
[3] data association filter", IEEE Control Systems Magazine Vol. 29, No. 6, PP. 82-100, December 2009.

[4] Blackman, Samuel, Robert Popoli, "Design and analysis of modern tracking systems", Boston: Artech House, 1999.

[5] Jaco Vermaak, Simon J. Godsill and Patrick P'erez, "Monte Carlo filtering for multi-target tracking and data association", IEEE Transactions On Aerospace And Electronic Systems Vol. 41, No. 1, 2005 , PP. 309-332

[6] G. W. Pulford "Multi-target Viterbi data association", Proc. of the $9^{\text {th }}$ International Conference on Information Fusion, Florence, Italy, July 2006.

[7] E.M.Saad, El.Bardawiny, H.I.ALI and N.M.Shawky, " Improving data association based on finding optimum innovation applied to nearest neighbor for multi-target tracking in dense clutter environment", IJCSI International Journal of Computer Science Issues, Vol. 8, Issue 3, No.1, PP.496-507, May 2011

[8] X. Wang, S. Challa, and R. Evans, "Gating techniques for maneuvering target tracking in clutter", IEEE Transactions on Aerospace and Electronic Systems, Vol..38, No.3, July 2002, PP. 1087-1097.

[9] G. David Forney, Jr. "The Viterbi algorithm". Proceedings of the IEEE, 61(3) , PP. 268-278, 1973.

[10] T. Quach and M. Farooq. "Maximum likelihood track formation with the Viterbi algorithm". In 33rd Conference on Decision and Control,CDC94, , Florida, USA, 1994, PP. 271-276.

[11] B. F. La Scala and G. W. Pulford. "Viterbi data association tracking for Over-The-Horizon Radar", In International Radar Symposium, IRS98, , Munich, Germany, 1998, PP. 1155-1164.

[12] Simon J. Julier and Je_rey K. Uhlmann. "A new extension of the Kalman filter to nonlinear systems", In Proc. AeroSense: 11th Int'l Symposium on Aerospace/Defense Sensing, Simulation and Controls, Orlando, Florida, 1997.

[13] E. A. Wan and R. van der Merwe, "The Unscented Kalman filter for nonlinear estimation", In Proceedings of IEEE Symposium 2000 on Adaptive Systems for Signal Processing, Communications and Control (AS-SPCC), Lake Louise, Canada, October 2000.

[14] Simon Haykin "Kalman filtering and neural networks" ,EBook,Published by JOHN WILEY \& SONS, INC.2001

[15] James V. Candy "Bayesian signal processing classical, modern, and particle filtering methods" ,E-Book, Published by JohnWiley \& Sons, New Jersey 2009.

[16] B. Ristic, S. Arulampalam, and N. Gordon," Beyond the Kalman filter,particle filters for tracking applications", Boston, MA: Artech House, 2004.

[17] M. Sanjeev Arulampalam, Simon Maskell, Neil Gordon, and Tim Clapp "A tutorial on particle filters for online nonlinear/non-gaussian bayesian tracking" IEEE Transactions on Signal Processing, Vol. 50, No. 2, PP $174-188$, Feb.2002.

[18] C. Hue, J.-P. Le Cadre, P. Pérez. "A particle filter to track multiple objects". In IEEE Workshop on Multi-Object Tracking, PP. 61-68, Vancouver, Canada, July 2001.

[19] A. Doucet, N. de Freitas, and N. Gordon," Sequential Monte Carlo methods in practice", New York: Springer, 2001.

[20] N. J. Gordon, D. J. Salmond, and A. F. M. Smith, "Novel approach to nonlinear/non-Gaussian Bayesian state estimation," Proc. Inst. Elect. Eng., Vol. 140, No. 2, pt. F, PP. 107-113, 1993.

[21] Jaward, M., Mihaylova, L., Canagarajah, N., \& Bull, D. "Multiple object tracking using particle filters". IEEE Aerospace Conference, 1-8. Institute of Electrical and Electronics Engineers, 2006

[22] YANG Xiaojuna, ${ }^{*}$, XING Keyib, FENG Xinglea" Maneuvering target tracking in dense clutter based on particle filtering", Chinese Journal of Aeronautics Elsevier Vol.. 24, PP. 171-180, 2011.

[23] Zia Khan, Tucker Balch, Member, IEEE, and Frank Dellaert, Member, IEEE, "MCMC-based particle filtering for tracking a variable number of interacting targets" IEEE Transactions on Pattern Analysis and Machine Intelligence, Vol.. 27, No. 11, PP. 1805-1819, Nov. 2005.

[24] Olivier Rabaste "Multi-target tracking with MCMC-based particle filters" 18th European Signal Processing Conference (EUSIPCO-2010) Aalborg, Denmark, August 23-27, 2010 , PP. 195-163.

[25] Hongtao Hu Zhongliang Jing Anping Li Shiqiang Hu Hongwei Tian "An MCMC-based particle filter for tracking target in glint noise environment "Proceedings The 7th International Conference on Information Fusion June 28 to July 1, 2004 in Stockholm, Sweden.

[26] Liu Jing, Prahlad Vadakkepat" Interacting MCMC particle filter for tracking maneuvering target" Elsevier Digital Signal Processing Vol. 20, No. 2, PP. 561-574, 2010.

[27] Franc,ois Septier, Sze Kim Pang, Avishy Carmi and Simon Godsill "On MCMC-based particle methods for Bayesian filtering: application to multitarget tracking" 2009 3rd IEEE International Workshop on Computational Advances in Multi-Sensor Adaptive Processing, , 2009, PP. 360-363.

[28] Gad.,Ahmed, and Farooq. M. "Single target tracking in clutter: performance comparison between PDA and VDA", proceeding of the sixth international conference of information fusion, Vol. .2 PP. 12661273, 2003.

[29] Ahmed Gad, F.Majdi M.Farooq "A comparison of data association techniques for target tracking in clutter", Proceedings of the Fifth IEEE International Conference on Information Fusion, Vol. 2, PP. 1126-1133, 2002.

[30] Y. Bar-Shalom and E. Tse, "Tracking in a cluttered environment with probabilistic date association", Automatica, Vol. 11, PP. 451-460, Sept. 1975. 